\title{
Circuit
}

Musiques contemporaines

\section{Björk Digital : escale à Montréal}

\section{Nicolas-Alexandre Marcotte}

Volume 27, numéro 2, 2017

URI : https://id.erudit.org/iderudit/1040880ar

DOI : https://doi.org/10.7202/1040880ar

Aller au sommaire du numéro

\section{Éditeur(s)}

Circuit, musiques contemporaines

\section{ISSN}

1183-1693 (imprimé)

1488-9692 (numérique)

Découvrir la revue

Citer ce document

Marcotte, N.-A. (2017). Björk Digital : escale à Montréal. Circuit, 27(2), 115-118. https://doi.org/10.7202/1040880ar

Ce document est protégé par la loi sur le droit d'auteur. L'utilisation des services d'Érudit (y compris la reproduction) est assujettie à sa politique d'utilisation que vous pouvez consulter en ligne.

https://apropos.erudit.org/fr/usagers/politique-dutilisation/
Cet article est diffusé et préservé par Érudit.

Érudit est un consortium interuniversitaire sans but lucratif composé de l’Université de Montréal, l'Université Laval et l'Université du Québec à Montréal. Il a pour mission la promotion et la valorisation de la recherche. https://www.erudit.org/fr/ 


\title{
Björk Digital : escale à Montréal
}

\author{
Nicolas-Alexandre Marcotte
}

C'est dans les locaux de la fondation DHC/ART ${ }^{1}$, dans le Vieux-Montréal, que prenait place en octobre 2016 l'exposition de réalité virtuelle (VR) de l'icône mondiale de la pop expérimentale Björk, à la suite des précédentes escales à Sydney, Tokyo et Londres. Après la sortie en 2011 de son septième album Biophilia, à partir duquel une application interactive avait été élaborée, l'artiste islandaise a visiblement souhaité poursuivre et approfondir les potentialités de résonances plus intimes, ou à tout le moins élargies, entre son œuvre et le public.

L'épine dorsale de Björk Digital consiste en la présentation de cinq expériences de réalité virtuelle construites autour d'autant de chansons extraites du dernier album de Björk, Vulnicura (2015). C'est par groupes d'une quinzaine de personnes qu'un parcours établi et encadré par le personnel de l'établissement est effectué à travers une succession d'espaces délimités, dans lesquels sont répartis un ensemble de tabourets pivotants, permettant une fois installés dessus d'apprécier les 360 degrés sphériques offerts par le casque de réalité virtuelle, sans risque de contact physique non désiré avec les autres «visiteurs ».

Si l'album Vulnicura est, d'après son auteure, né d'une rupture amoureuse ${ }^{2}$ et en décline les étapes émotionnelles subséquentes - Björk affirme qu'il s'agit du premier de ses disques dont l'ordre des chansons tient compte d'une chronologie précise ${ }^{3}-$, on souscrira plus facilement au fait que chacune de ces expériences est entièrement polarisée sur la personne de Björk.

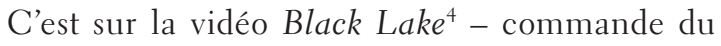
Museum of Modern Art de New York et réalisée par le cinéaste Andrew Thomas Huang - que s'ouvre le par-

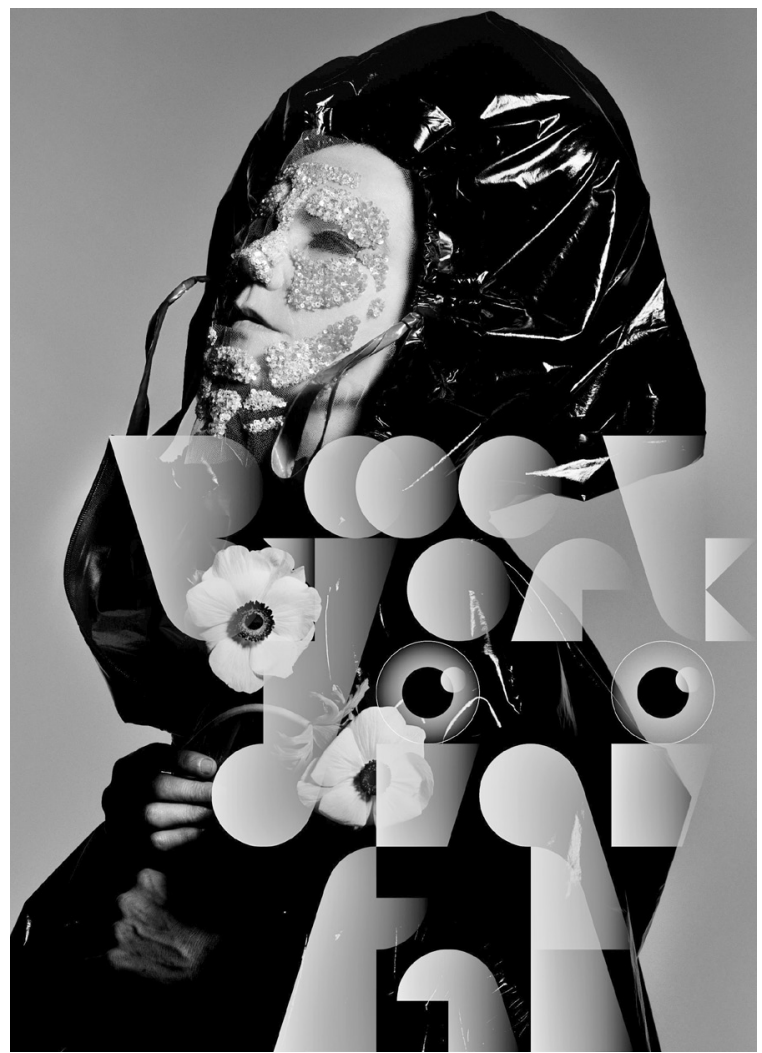

cours immersif, où l'on se voit positionné à l'intérieur d'une caverne sombre. La sobriété du lieu s'avère en concordance avec la trame musicale épurée : un quatuor à cordes aux accents mélancoliques soutenu sporadiquement par une rythmique électronique sourde et lointaine. C'est dans ce lieu où la symbolique de l'enfermement et du repli est manifeste que Björk apparaît simultanément projetée sur différentes parois (au gré de l'angle adopté par le spectateur), telle une peinture rupestre en mouvement, s'ébattant avec une fougue qui n'est pas sans rappeler l'émoi fataliste de 
Selma Jezkova ${ }^{5}$ dans le film Dancer in the Dark, de Lars Van Triers.

Si cette entrée en la matière nous laisse plutôt l'impression d'être cantonné au rôle de simple observateur, elle semble de fait davantage consister en un refaçonnage - ou une forme d'extension technologisée - d'un vidéoclip préalablement destiné à l'écran traditionnel qu'un objet élaboré sur mesure pour la réalité virtuelle.

Les choses prennent toutefois une autre tournure avec l'œuvre Stonemilker ${ }^{6}$ - également signée A.T. Huang - où, sortant du confinement quelque peu oppressant de la première expérience, nous nous retrouvons au milieu du paysage dégagé et venteux d'un bord de mer islandais. Vêtue de jaune vif contrastant avec la grisaille ambiante (avec l'annonce au loin d'une tempête à venir?), une Björk démultipliée apparaît et disparaît incessamment autour de nous, nous tendant les mains en une imploration chargée d'émotion. Chanson sur le thème de la déconnexion malgré la proximité, l'aspect immersif de la réalité virtuelle sied ici assez bien, nous obligeant à nous mouvoir sur le tabouret pour tenter de garder un contact visuel avec la chanteuse, voire quasiment pouvoir la toucher. Le contraste entre l'immensité de la scène et les (éphémères) tentatives de rapprochement teintées d'intimité est musicalement réverbéré par une partition où le quatuor à cordes, fil conducteur instrumental de l'album, se voit également démultiplié en un ensemble plus imposant et accompagné d'une rythmique tantôt stellaire, tantôt souterraine.

La vidéo suivante, Mouth Mantra ${ }^{7}$, nous amène dans une dimension qui sera résolument la plus abstraite du parcours soit, littéralement, l'intérieur de la bouche de Björk. Chanson sur l'impuissance à se faire entendre, à se faire comprendre, le réalisateur Jesse Kanda scrute impudiquement les mouvements organiques de l'appareil buccal de la chanteuse, captés à l'aide de plusieurs micro-caméras qui y ont été introduites. La sensation de voyeurisme combinée avec l'extrême proximité de cette partie interne de la physiologie humaine se mêle au malaise, au sentiment d'oppression exprimé par Björk, rendant cette expérience la plus - volontairement? - inconfortable de l'exposition. La trame musicale s'avère en phase avec cet état d'esprit, où le traitement électronique de la voix fragmente une ligne mélodique déjà fortement scandée et déconstruite.

L'avant-dernière présentation est le fruit d'une étroite collaboration avec le Dentsu Lab Tokyo ${ }^{8}$ et résulte d'une captation en "réalité virtuelle augmentée » d'une prestation de Björk au musée Miraikan de Tokyo. Hymne à l'univers, à la totalité, à la fertilité, Quicksand nous extirpe de la torpeur de Mouth Mantra sur une musique aérienne où un dédoublement légèrement décalé de la voix de la chanteuse semble vouloir nous rappeler de manière subliminale la pluralité de l'existence. Visuellement, dans un environnement induisant l'impression de flotter dans un espace indéfini, on retrouve l'artiste arborant une coiffe - créée par imprimante $3 \mathrm{D}$ par l'artiste Neri Oxman ${ }^{9}$ - d'où semblent émerger des formes d'énergies éthérées, lumières irréelles et champs magnétiques colorés. Quicksand combine ici une réalité à mi-chemin entre le monde réel et palpable - il s'agit d'une prestation de la chanteuse dont les mouvements sont filmés «en direct» dans un lieu physique défini (la salle du musée Miraikan) - et le monde virtuel et imaginaire (par les nombreux effets visuels informatisés qui y sont insérés), et constitue en quelque sorte le tremplin vers l'apothéose du parcours, Family.

Seule expérience réellement interactive de l'exposition, Family ${ }^{10}$ est également l'unique ouvre entièrement élaborée sur une base purement virtuelle, soit par images de synthèse, à la manière de jeux vidéo. Aboutissement de trois années de collaboration entre A.T. Huang et le directeur artistique James Merry, il s'agit d'une certaine façon de la conclusion naturelle de Vulnicura, dont le titre provient du latin vulnus, 
«blessure", et cura, "soin", où le spectateur - cette fois non plus assis en groupe, mais debout, isolé dans un espace circonscrit par des rideaux -, après avoir été le témoin impuissant de la déroute émotionnelle de la chanteuse, adopte soudainement le rôle de "guérisseur ». Muni d'un dispositif permettant de mouvoir des mains virtuelles, c'est à l'aide de celles-ci qu'il est appelé à soigner la blessure métaphysique - au moyen de rubans colorés expulsés à la pression d'un bouton - d'une irréelle et luminescente Björk, au son d'incantations solitaires, démultipliées et harmonisées par vocodeur, où l'absence d'armature rythmique de la trame d'accompagnement amplifie le caractère intemporel et féérique du moment.

En marge des cinq expériences de réalité virtuelle était également présentée l'application pour tablette électronique Biophilia ${ }^{11}$, élaborée en jonction avec le précédent album éponyme de Björk et ayant donné naissance au Biophilia Educational Project ${ }^{12}$. Toute première application électronique (app) faisant son entrée officielle dans la collection du Museum of Modern Art de New York, cet ambitieux ouvrage conçu par une équipe internationale de créateurs ${ }^{13}$ - se veut une porte d'entrée à la fois ludique et créative vers la création sonore via dix «mini-applications », en correspondance aux dix chansons de l'album. Avec pour intention d'établir un pont tactile et palpable entre le son, de nature invisible, et différents objets et matériaux constituants de l'univers (chaîne d'ADN, cellules, système solaire, ondes lumineuses, matière sombre, etc.), la nature interactive de ces créations, situées à michemin entre le jeu et l'instrument de musique, permet ici de modifier nombre de paramètres sonores et musicaux (hauteurs, ordre des notes, rythmes, durées, intensités, densité harmonique, forme) et d'entrer dans un processus de déconstruction/reconstruction et donc de recréation des chansons originales de Björk.

L'exposition Björk Digital se concluait avec la présentation d'une sélection de 30 vidéoclips réalisés au cours de la carrière de la chanteuse sur une période de 24 années. Extension presque incontournable aux chansons dans le monde de la musique populaire et outil commercial à la valeur artistique parfois discutable, le vidéoclip s'est tout de même vu acquérir des lettres de noblesse grâce au travail sérieux de réalisateurs reconnus pour renouveler le langage visuel et cinématographique par des techniques souvent audacieuses. Michel Gondry ${ }^{14}$ et Spike Jonze ${ }^{15}$ sont notamment de ceux qui ont su développer une étroite collaboration avec Björk et offrir une synthèse artistique à l'image de la chanteuse, dont l'une des principales caractéristiques est le goût prononcé pour les collaborations extérieures ainsi que l'inclination à absorber et se réapproprier les tendances les plus diverses, tant sur le plan musical que des arts visuels et de la mode.

On ne peut qu'admettre que le médium du vidéoclip semble pour Björk manifester la volonté de dépasser le cadre de simple extension visuelle aux œuvres musicales et illustre le besoin de l'artiste de déployer un ambitus d'expression plus entier, universel, chargé de références et de symboles. Lutilisation des technologies de réalité virtuelle dans Vulnicura semble présenter une nature et une affectation propres à dépasser le rôle de simple valeur ajoutée à l'image bidimensionnelle, ouvrant une nette voie - en plus d'une immersion accrue - à l'implication active du spectateur ${ }^{16}$ dans un genre qui, malgré tout, a encore beaucoup à prospecter afin de se positionner de manière distinctive face au modèle du jeu vidéo.

\footnotetext{
1. Voir: http://dhc-art.org/fr (consulté le 24 mai 2017).

2. Björk s'est séparée de l'artiste multidisciplinaire Mathew Barney en 2013. Voir: Brigid Delaney (2016), "Björk: 'It's No Coincidence that the Porn Industry Has Embraced Virtual Reality" ", The Guardian, https://www.theguardian.com/music/2016/jun/o3/bjork-its-nocoincidence-that-the-porn-industry-has-embraced-virtual-reality (consulté le 15 juin 2017).
} 
3. C'est après avoir écrit les chansons que Björk affirme s'être rendu compte d'avoir tissé, sans le savoir, la trame narrative d'une tragédie grecque. Voir: Brigid Delaney, loc. cit.

4. Voir: www.youtube.com/watch?v=YGn1pJIpZw8 (consulté le 24 mai 2017).

5. Nom du personnage principal incarné par Björk dans ce long métrage paru en 2000 et qui remporta la Palme d'or au $53^{\mathrm{e}}$ Festival de Cannes.

6. Voir: www.youtube.com/watch?v=gQEyezu7G20 (consulté le 24 mai 2017). Si le disque présuppose un ordre chronologique des pièces, il est étrange de remarquer qu'il n'est pas suivi dans le cadre de l'exposition, Stonemilker étant la première pièce figurant sur l'album et semblant donc préluder au sein de la trame narrative la rupture de Björk.

7. Voir: www.youtube.com/watch?v=ilhLCXmrCm8 (consulté le 24 mai 2017).

8. Le Dentsu Lab Tokyo se veut une équipe de réflexion créative combinant recherche et développement à travers les nouvelles technologies. II œuvre tant dans le domaine de la publicité que des arts de la scène et des productions télévisuelles et cinématographiques. Un de leurs plus récents projets est une collaboration avec le musicien Brian Eno. Voir: http://dentsulab.tokyo (consulté le 24 mai 2017).

9. Sur Neri Oxman, voir: www.materialecology.com (consulté le 24 mai 2017).

10. Voir: www.youtube.com/watch?v=wMCOCvrFJgw et www. youtube.com/watch?v=HAXvkbOzK6E (consultés le 24 mai 2017).
11. La biophilie est un néologisme inventé par Edward O. Wilson se définissant comme un "penchant naturel, instinctif, qui nous pousse à nous intéresser à la vie et à ses processus, et rechercher un contact, authentique ou dérivé, mais intime, avec le vivant dans ses différents niveaux d'expression " (www.humanite-biodiversite.fr/article/ biophilie, consulté le 24 mai 2017).

12. Initialement développé par Björk Guđmundsdóttir, la ville de Reykjavik et l'Université d'Islande, le Biophilia Educational Project réunit la majorité des pays scandinaves et vise à inspirer les enfants de dix à douze ans à explorer leur créativité à travers l'apprentissage de la musique, de la nature, des sciences et des nouvelles technologies. Voir: http://biophiliaeducational.org (consulté le 24 mai 2017).

13. Mathias Augustyniak et Michael Amzalag de M/M Paris, Sjón, Scott Snibbe, Sarah Stocker et Mark Danks de Kodama Studios, Touch Press, Max Weisel de Relative Wave, Nikki Deben, Stephen Malinowski et John F. Simon, Jr.

14. À titre de réalisateur de vidéoclips, Michel Gondry est notamment connu pour ses nombreuses collaborations avec les Rolling Stones. Il s'est également retrouvé derrière la caméra pour le long métrage Eternal Sunshine of the Spotless Mind (2004), ainsi que pour le documentaire An Animated Conversation with Noam Chomsky (2013).

15. Spike Jonze a notamment réalisé des vidéoclips pour The Chemical Brothers, R.E.M., The Beastie Boys et Arcade Fire. II est également le réalisateur des longs métrages Being John Malkovitch (1999) et Her (2013).

16. Nous faisons ici référence à Family, la "pièce maîtresse" de l'exposition.

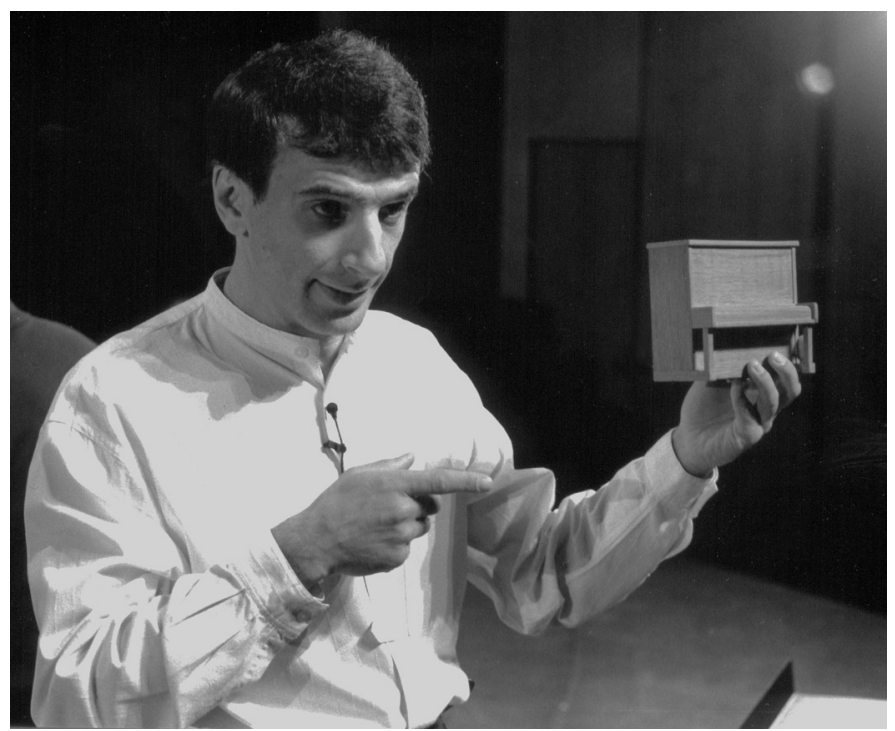

Jacques Piperni dans la première version du spectacle jeunesse Le piano muet (livret de Gilles Vigneault, musique de Denis Gougeon), 1996. 